BULL. AUSTRAL. MATH. SOC.

VOL. $21(1980), 407-417$.

\title{
THE COMPLETION OF A TOPOLOGICAL GROUP
}

\author{
Eric C. Nummela
}

\begin{abstract}
During the 1920's and 30's, two distinct theories of
"completions" for topological spaces were being developed: the French school of mathematics was describing the familiar notion of "complete relative to a uniformity", and the Russian school the less well-known idea of "absolutely closed". The two agree precisely for compact spaces.

The first part of this article describes these two notions of completeness; the remainder is a presentation of the interesting, but apparently unrecorded, fact that the two ideas coincide when put in the context of topological groups.
\end{abstract}

\section{The completion of a topological space}

What does it mean to say that a topological space $X$ is complete? (For convenience, we will assume that all topological spaces satisfy the Hausdorff separation axiom.) The reader's answer to this question in the case where $X$ is a metric space would probably be:

(A) $X$ is a complete metric space if and only if every Cauchy sequence converges.

If (A) is taken as a correct answer, another familiar property of complete metric spaces can be expressed as follows:

(B) a complete subspace $Y$ of a metric space $X$ is closed in $X$ (whether $X$ is complete or not).

Received 27 November 1979. 
The study of topology was at first largely confined to metric spaces. Just as Cantor completed the rational numbers, so can an arbitrary metric space $X$ be completed by means of Cauchy sequences. The completion $\hat{X}$ contains $X$ as a dense subspace, and the metric on $\hat{X}$ extends the metric on $X$. The first thorough treatment of metric spaces was given by Hausdorff in 1914 [11].

Unfortunately, statement (A) depends on the metric on $X$; that is, $X$ may admit two metrics compatible with its topology, and be complete relative to one but not the other. Consider the following example.

EXAMPLE 1. Let $d_{1}$ denote the usual metric on $R$, the set of real numbers; that is, $d_{1}(x, y)=|x-y|$. Then neither $Q$, the rationals, nor $I$, the irrationals, is closed in $R$; hence by (B) neither is $d_{1}$-complete. Following [15, p. 59], we define another metric $d_{2}$ on $R$ as follows: let $\left\{r_{i}\right\}$ be an enumeration of $Q$, and set

$$
d_{2}(x, y)=|x-y|+\sum_{i=1}^{\infty} 2^{-i} \inf \left\{1,\left|\max _{j \leq i} \frac{1}{\left|x-r_{j}\right|}-\max _{j \leq i} \frac{1}{\left|y-r_{j}\right|}\right|\right\} .
$$

Then $d_{2}$ adds to the $d_{1}$-distance between $x$ and $y$ an amount less than or equal to one which somehow reflects the relative distances of $x$ and $y$ from the rationals $\left\{r_{i}\right\}$. If $B_{i}(p, \varepsilon)$ (for $i=1,2$ ) is the open $d_{i}$-ball of radius $\varepsilon$ about the point $p$, then it is clear that $B_{2}(p, \varepsilon) \subseteq B_{1}(p, \varepsilon)$. Thus the $d_{2}$-topology on $R$ is finer than the $d_{1}$-topology

The converse fails, however. Note first that if $p$ is rational and $\varepsilon$ is sufficiently small, then $B_{2}(p, \varepsilon)=\{p\}$. Thus $Q$ is $d_{2}$-open in $R$, and the $d_{2}$-topology restricted to $Q$ is discrete. Second, if $p$ is irrational and $\varepsilon$ is given, then we can always find $\delta$ such that $B_{1}(p, \delta) \subseteq B_{2}(p, \varepsilon)$. Thus $d_{2}$ induces on $I$ the same topology as $d_{1}$.

Finally, note that $I$ is $d_{2}$-complete. Any $d_{2}$-Cauchy sequence $\left\{x_{n}\right\}$ in $I$ is $d_{1}$-Cauchy, and hence $d_{1}$-converges to some $x$ in $R$. If $x=r_{k}$ for some $k$, then for any $n$ there exists $m>n$ such that 
$d_{2}\left(x_{n}, x_{m}\right) \geq\left|x_{n}-x_{m}\right|+2^{-k}$ - a contradiction. Thus $x \in I$; and thus $\left\{x_{n}\right\} d_{2}$-converges to $x$. That is, $I$ is $d_{2}$-complete. (On the other hand, since $Q$ with its usual topology is not a Baire space, no metric compatible with that topology can be complete [6, IX,5.3, Theorem 1].

In 1924, the Russian school of mathematics took a different view of "completeness". In a fundamental article [3], Alexandroff and Urysohn defined compact spaces. The fact that a compact subspace $K$ of a topological space $X$ is always closed in $X$ is analogous to statement (B), and seems to have motivated the definition, also in [3], of absolutely closed space. A topological space is said to be absolutely closed provided it is closed in every extending space. Since every completely regular space admits a compactification, an absolutely closed space which is completely regular must be compact. In particular, an absolutely closed metric space is compact. The following theorem characterizes absolutely closed spaces.

THEOREM 2. A space is absolutely closed if and only if it is almost compact, which means that any collection of open sets which covers the space has a finite subcollection whose closures cover the space [3, Satz II].

Proof. Assume $X$ is absolutely closed, let $U=\left\{U_{i}\right\}_{i \in I}$ (where $I$ is an arbitrary index set) be an open cover of $X$, and assume no finite subcollection of closures of elements of $u$ covers $X$. Then, for each finite subset $F \subseteq I$, the set $U_{F}=X-\bigcup_{i \in F} \bar{U}_{i}$ is a non-empty open subset of $X$. Adjoin a new point $p$ to $X$, and let

$$
\left\{U_{F} \mid F \text { is a finite subset of } I\right\}
$$

be a fundamental system of neighborhoods of $p$. Then $X \cup\{p\}$ is a (Hausdorff) topological space extending $X$, and $X$ is not closed in $X \cup\{p\}$. This contradiction shows that for some $F, X=\cup_{i \in F} \bar{U}_{i}$.

Conversely, assume $X$ is almost compact, assume $X$ is a subspace of the space $Y$, and let $p \in Y-X$. Then for each $x \in X$ we can find a neighborhood $U_{x}$ of $x$ in $y$ such that $p \notin \bar{U}_{x}$. Since $\left\{X \cap U_{x}\right\}$ is an 
open cover of $X$, some finite subcollection satisfies the property that their closures in $X$ cover $X$; hence their closures in $Y$ cover $X$. But $p$ is not in the union of this finite collection of closed sets. Thus $X$ is closed in $Y$; and hence $X$ is absolutely closed.

That absolutely closed spaces may be non-compact is shown by the following example.

EXAMPLE 3 [15, pp. 119-120]. Let $A$ be the linearly ordered set $\{1,2,3, \ldots, \omega, \ldots,-3,-2,-1\}$ with the interval topology, and let $z^{+}$ be the set of positive integers with the discrete topology. We define $x$ to be $A \times Z^{+}$together with two ideal points $a$ and $-a$. The topology on $X$ is determined by the product topology on $A \times Z^{+}$together with basis neighborhoods $M_{n}^{+}(\alpha)=\{a\} \cup\{(i, j) \mid i<\omega$ and $j>n\}$ and $M_{n}^{-}(-a)=\{-a\} \cup\{(i, j) \mid i>\omega$ and $j>n\}$. Then $X$ is a Hausdorf topological space; however, $X$ is not regular, since for all positive integers $m$ and $n$,

$$
\overline{M_{m}^{+}(a)} \cap \overline{M_{n}^{-}(-a)}=\{(\omega, i) \mid i>\max (m, n)\} \neq \emptyset \text {. }
$$

Hence $X$ is not compact. Nevertheless, $X$ is almost compact, for any collection of open sets which covers $X$ must contain open sets containing $a$ and $-a$, and the closures of these two open sets contain all but finitely many of the points $(\omega, j)$.

In 1939, Alexandroff [2] showed that if $X$ is a regular space, then $X$ has a universal absolute closure; that is, there exists an absolutely closed regular space $X^{*}$ which contains $X$ as a dense subspace and such that any continuous function from $X$ into an absolutely closed regular space has a continuous extension to $X^{*}$ [2]. For an elegant exposition of Alexandroff's work, as well as a partial (and best possible) extension to arbitrary spaces, see [10]. An excellent expository treatment of the general problem of determining extensions of topological spaces is given in [4].

Also in the late 1930's there were two developments in the French school of mathematics that served to extend the metric notion of completeness to a much larger class of spaces. One was the invention of filters by Cartan [7], [8]. A filter on a set $X$ is a collection of 
subsets of $X$ which is closed with respect to supersets and finite intersections. For example, the set of all neighborhoods of a point in a topological space is a filter, called the neighborhood filter of the point. (A neighborhood of $x$ need not be open, but it must contain an open subset containing $x$.) Filters provide a generalization of sequences to arbitrary topological spaces: a filter is said to converge to a point $x$ if it is contained in the neighborhood filter of $x$.

The second development was the invention of uniform spaces by Weil [16]. The presence of a metric on a space $X$ provides a way of comparing the "closeness", one to another, of any pair of points of $X$. But, as recognized by Weil, the properties of closeness can be specified fully as well by a suitably chosen collection of subsets of $X \times X$, each containing the diagonal $\Delta$. More specifically, a uniformity $\mu$ on a set $X$ is a filter of subsets, called entourages, of $X \times X$ satisfying the following conditions:

(1) for each $U \in \mu, \Delta \subseteq U$;

(2) if $U \in \mu$, then $U^{-1}=\{(x, y) . \mid(y, x) \in U\} \in \mu$;

(3) for each $U \in \mu$, there exists $V \in \mu$ such that

$V \circ V=\{(x, y) \mid$ there exists $z \in X$ with $(x, z),(z, y) \in V\} \subseteq U$;

(4) $\cap\{U \mid U \in \mu\}=\Delta$.

All pairs of points belonging to the same entourage satisfy the same "closeness" property. A uniformity $\mu$ on $X$ induces a topology on $X$ by taking for the neighborhood filter of $x \in X$ the collection of subsets $\{U(x) \mid U \in \mu\}$, where $U(x)=\{y \mid(x, y) \in U\}$. Note that it is condition (4) above which guarantees that such a topology is Hausdorff. Weil proved that the class of uniform spaces coincides with the class of completely regular spaces, and thus contains all compact spaces and all metric spaces.

A filter $F$ on a uniform space $X$ is a Cauchy filter if, given any entourage $U$, there exists $F \in F$ with $F \times F \subseteq U$. (If we think of $U$ as being a measure of "closeness", then any pair of points from $F$ are "close" together.) Every convergent filter on $X$ is Cauchy; and if every Cauchy filter on $X$ converges, then $X$ is complete. Just as for metric spaces, every uniform space $X$ can be embedded as a dense subspace of a 
complete uniform space $\hat{X}$, and the uniformity of $\hat{X}$ extends the uniformity of $X$ [6, II, \$3.7]. Moreover, $\hat{X}$ is universal; that is, every uniformly continuous function from $X$ to a complete uniform space has a uniformly continuous extension to $\hat{X}$. (Of course, a function $f: X \rightarrow X^{\prime}$ of uniform spaces is uniformly continuous if its behavior with respect to entourages is analogous to the behavior of a continuous function with respect to neighborhoods; that is, given any entourage $V^{\prime}$ of $X^{\prime}$, there exists an entourage $V$ of $X$ such that $(x, y) \in V$ implies $\left.(f(x), f(y)) \in V^{\prime}.\right)$

Although we have seen that the question at the beginning of this section is ambiguous, we shall see in the next section that the ambiguity vanishes in the context of topological groups.

\section{The completion of a topological group}

The first comprehensive approach to completing topological groups was suggested by Weil. While he was laying the foundations of the theory of uniform spaces [16], he noted that there are two natural ways of describing a uniform structure on a topological group $G$ : the elements $x$ and $y$ of $G$ are said to be $V$-close, where $V$ is a neighborhood of the identity $e$ in $G$, if $y$ is carried into $V$ by translation by $x^{-1}$. Since multiplication in $G$ is, in general, not commutative, we should expect to obtain two notions of $V$-closeness, depending on whether we multiply $y$ by $x^{-1}$ on the right or on the left. More specifically, we denote by $V_{d}$ (respectively $V_{s}$ ) the set of all $(x, y)$ in $G \times G$ such that $y x^{-1} \in V$ (respectively, $x^{-1} y \in V$ ). Then as $V$ runs through a fundamental system of neighborhoods of $e$, the sets $V_{d}$ (respectively $V_{s}$ ) describe a fundamental system of entourages of the right (respectively left) uniformity of $G$. Each uniformity is compatible with the topology of $G$, and in general the two uniformities are distinct. (Obviously, the uniformities coincide if $G$ is abelian; they also coincide if $G$ is compact, since a compact space admits only one uniformity compatible with its topology. An interesting characterization of groups with equal uniformities is given in terms of nets by Yang [18].) Weil also proved that every continuous homomorphism of topological groups is both right and 
left uniformly continuous (see [6, III, §3.1]).

Although Weil considered the problem of completing a topological group relative to its right (or left) uniformity, definitive results were not published until 1942, when Bourbaki proved that a topological group $G$ is isomorphic to a dense subgroup of a complete (relative to either the right or left uniformity) group if and only if every right Cauchy filter is also a left Cauchy filter [5]. Bourbaki then asked if every topological group satisfied this latter property [5, p. 28]. Dieudonné showed in 1944 that the answer to Bourbaki's question was negative [9]. We present his example below.

EXAMPLE 4. Let $I$ denote the unit interval $[0,1]$, and let $G$ be the group of homeomorphisms of $I$ onto itself. (If $u, v \in G$, then $u v$ denotes the composite homeomorphism $x \mapsto u(v(x))$, and $u^{-1}$ denotes the inverse of $u$.) Endow $G$ with the topology of uniform convergence on $I$, or what amounts to the same thing, the topology defined by the metric $\sup |u(x)-v(x)|$. It is easily verified that this topology is compatible $x \in I$

with the group structure of $G$; that is, $G$ is a topological group. Since $G$ is a metric group, we allow ourselves to use Cauchy sequences in place of Cauchy filters. A sequence $\left\{u_{n}\right\}$ in $G$ is right (respectively left) Cauchy if and only if for any $\varepsilon>0$ there exists $N$ such that $n, m>N$ implies $\left|u_{n} u_{m}^{-1}(x)-x\right| \leq \varepsilon$ (respectively, $\left|u_{m}^{-1} u_{n}(x)-x\right| \leq \varepsilon$ ) for all $x \in I$. Now consider the sequence $\left\{u_{n}\right\}$, where $u_{n}$ is the homeomorphism which is equal to 0 if $x=0$, to 1 if $x=1$, to $I / n$ if $x=\frac{3}{2}$, and linear for $0 \leq x \leq \frac{1}{2}$ and $\frac{1}{2} \leq x \leq 1$. Then $\left\{u_{n}\right\}$ is a right Cauchy sequence - the details are messy, but straightforward. On the other hand, $\left\{u_{n}\right\}$ is not a left Cauchy sequence. To see this, note that if $x=\frac{3}{2}$ and $m>n$, then

$$
\left|u_{n}^{-1} u_{m}(x)-x\right|=\left|u_{n}^{-1}(1 / m)-\frac{1}{2}\right|=\left|(n / 2 m)-\frac{1}{2}\right|=|(m-n) / 2 m| \text {. }
$$

Now, regardless of how large $N$ is, we take $n$ fixed (with $n>N$ ) and let $m \rightarrow \infty$. Then $|(m-n) / 2 m| \rightarrow \frac{l}{2}$. If $\left\{u_{n}\right\}$ were left Cauchy, then by taking $N$ sufficiently large, we could always make $|(m-n) / 2 m|$ as small as we please; since this is not the case, $\left\{u_{n}\right\}$ is not left Cauchy. 
Only as an exercise did Bourbaki note that every topological group is completable relative to its two-sided uniformity, which is the smallest uniformity containing both the right and left uniformities (see [5, III, $\S 3$, Ex. 6], or the same exercise in [6]). Further, the two-sided completion is universal. That is, let $G$ and $G^{\prime}$ be topological groups, and let $f: G \rightarrow G^{\prime}$ be a continuous homomorphism. Since $f$ is both right and left uniformly continuous, $f$ is uniformly continuous relative to the two-sided uniformities on $G$ and $G^{\prime}$. Thus if $G^{\prime}$ is complete relative to its two-sided uniformity, then $f$ extends uniquely to a continuous homomorphism from the two-sided completion of $G$ into $G^{\prime}$.

Meanwhile, the Russians were applying the notion of absolutely closed to topological groups. In 1942, A.D. Alexandroff noted that every topological group can be densely embedded in an absolutely closed topological group [1]. Of course, by an absolutely closed topological group, Alexandroff meant a topological group which is closed in every extending topological group. In 1946, Raikov gave a comprehensive account of "completions" of topological groups, via the concept of "funnel" [13]. Raikov's funnels are simply filters which are both right and left Cauchy; thus his completions are two-sided completions. Yet he identified his complete groups with Alexandroff's absolutely closed groups, and did not mention uniformities explicitly. (On the other hand, Bourbaki did not mention absolutely closed groups.) Moreover, Raikov proved the universality of the absolute closure of a topological group. That is, every continuous homomorphism from a topological group $G$ into an absolutely closed topological group $G^{\prime}$ extends uniquely to a continuous homomorphism from the absolute closure of $G$ into $G^{\prime}$.

Finally, several authors have noted that in the context of metrizable topological groups, absolutely closed is equivalent to complete in the two-sided uniformity (see [12], [14], [17], and the review of [17] in Mathematical Reviews).

Thus there seems to be no good reason for the following theorem not to have been stated explicitly before now, except for the disparate vocabulary of the Russian and French schools of mathematics. The proof we provide is based on the universality of both completions and absolute closures.

THEOREM 5. Let $G$ be a topological group, let $G^{*}$ be the absolute 
closure of $G$, and let $\hat{G}$ be the two-sided completion of $G$. Then $G^{*}$ and $\hat{G}$ are isomorphic as topological groups, via an isomorphism fixing the elements of $G$.

Proof. Since $G^{*}$ is both closed and dense in $\left(G^{*}\right)^{\wedge}$, we have $G^{*}=\left(G^{*}\right)^{\wedge}$; that is, $G^{*}$ is complete in its two-sided uniformity. Hence the inclusion $G \rightarrow G^{*}$ extends uniquely to a continuous homomorphism $f: \hat{G} \rightarrow G^{*}:$

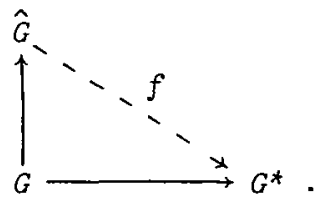

Also, $\hat{G}$ is dense in $(\hat{G}) *$. Since the two-sided uniformity of $(\hat{G}) *$ extends the two-sided uniformity of $\hat{G}$, with respect to which $\hat{G}$ is complete, then $\hat{G}$ is closed in $(\hat{G})^{*}$, and hence $\hat{G}=(\hat{G})^{*}$; that is, $\hat{G}$ is absolutely closed. Hence the inclusion $G \rightarrow \hat{G}$ extends uniquely to a continuous homomorphism $g: G^{*} \rightarrow \hat{G}$ :

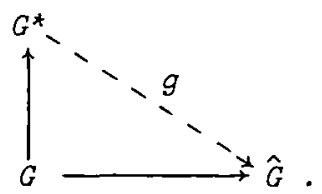

Now $f g: G^{*} \rightarrow G^{*}$ extends the inclusion $G \rightarrow G^{*}$, and so does the identity homomorphism on $G^{*}$. By uniqueness, then, $f g$ is the identity. Similarly, $g f$ is the identity homomorphism on $\hat{G}$. Thus we have the desired isomorphism.

Thus for any topological group $G$, the two-sided completion of $G$, which always exists and is absolutely closed, deserves to be called the completion of $G$.

\section{References}

[1] A.D. Alexandroff, "On the extension of a Hausdorff space to an H-closed space", C.R. (DokZ.) Acad. Sci. URSS (N.S.) 37 (1942), $118-121$. 
[2] П. Аленаңдров [P. Alexandroff], "Еиномпантные расшнрения топологичесних пространсвах Рауме" [Bikompakte Erweiterungen topologischer Räume], Rec. Math. [Mat. Sb.] (N.S.) 5 (47) (1939), 403-423.

[3] Paul Alexandroff und Paul Urysohn, "Zur Theorie der topologischen Räume", Math. Ann. 92 (1924), 258-266.

[4] Bernhard Banaschewski, "Extensions of topological spaces", Canad. Math. Buzz. 7 (1964), 1-22.

[5] N. Bourbaki, Élẻments de mathématique. Part I. Les structures fondamentales de l'analyse. Livre III. Topologie générale. Chapitres III et IV (Actual. Sci. Ind., 916. Hermann, Paris, 1942).

[6] Nicolas Bourbaki, Elements of mathematics. General topology, Parts 1 and 2 (Hermann, Paris; Addison-Wesley, Reading, Massachusetts; Palo Alto; London; 1966).

[7] Henri Cartan, "Théorie des filtres", C.R. Acad. Sci. Paris 205 (1937), 595-598.

[8] Henri Cartan, "Filtres et ultrafiltres", C.R. Acad. Sci. Paris 205 (1937), 777-779.

[9] Jean Dieudonné, "Sur la complétion des groupes topologiques", C.R. Acad. Sci. Paris 218 (1944), 774-776.

[10] S. Fomin, "Extensions of topological spaces", Ann. of Math. (2) 44 (1943), 471-480.

[11] Felix Hausdorff, Grundzüge der Mengenlehre (Veit, Leipzig, 1914. Reprinted: Chelsea, New York, 1949).

[12] V.L. Klee, Jr., "Invariant metrics in groups (Solution of a problem of Banach)", Proc. Amer. Math. Soc. 3 (1952), 484-487.

[13] Д. Раннов [D. Raikov], "О пополнение топологичссних групп" [On the completion of topological groups", Bull. Acad. Sci. URSS Sér. Math. [Izv. Akad. Nauk SSSR] 10 (1946), 513-528.

[14] Z. Semadeni, "Sur les groupes métriques complets", Colzoq. Math. 7 (1959), 35-39. 
[15] Lynn A. Steen, J. Arthur Seebach, Jr., Counterexcomples in topology (Holt, Rinehart and Winston, New York, Montreal, London, 1970).

[16] André Weil, Sur les espaces à structure uniforme et sur Za topologie générale (Actualités Scientifiques et Industrielles, 551. Hermann, Paris, 1937).

[17] J.D. Weston, "Incomplete subspaces of a Hausdorff space", Arch. Math. 10 (1959), 40-41.

[18] J.S. Yang, "A note on uniform structures of topological groups", Amer. Math. Monthly 79 (1972), 383-385.

Department of Mathematics,

New England College,

Henniker,

New Hampshire 03242,

USA. 